\title{
An Associationist Bias Explains Different Processing Demands for Toddlers in Different Traditional False-Belief Tasks
}

\author{
Marco Fenici ${ }^{a}$ Duilio Garofoli ${ }^{b}$

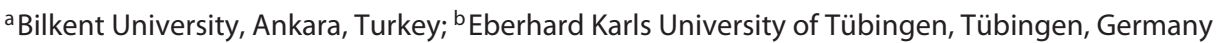

A nativist perspective regarding understanding others' mental states (Baillargeon, Scott, \& Bian, 2016) predicts that children should succeed at traditional false-belief tasks (FBTs, Wimmer \& Perner, 1983) before the age of 4 if their processing demands are appropriately reduced. To test this hypothesis, Setoh, Scott, and Baillargeon (2016; see also Grosso, Schuwerk, Kaltefleiter, \& Sodian, 2019) presented toddlers with a modified low-processing demand task (LPDT), in which a fictional character, Emma, finds an apple in a bowl, moves it to a lidded box, and then leaves the room. While she is away, her brother, Ethan, finds the apple and takes it away. When Emma returns, the child is asked: "Where will Emma look for her apple?"

The LPDT significantly reduces the inhibitory-control demands of traditional FBTs because the target object is not present on the scene during the final question (Wellman, Cross, \& Watson, 2001). Furthermore, the authors interspersed the task scenario with two practice trials in which children were familiarized with "where" questions to lower the executive demands of the test final question.

Setoh et al. (2016, experiment 1) found that, when both modifications were simultaneously included, even 30 -month-olds were significantly above chance in passing the task. In contrast, only 33 - but not 30 -month-olds' performance was above chance when the two trial questions did not contrast the correct object against an alter-

karger@karger.com

(c) 2020 S. Karger AG, Basel

www.karger.com/hde

Karger" native (experiment 2). Moreover, even 33-month-olds answered at chance if given only one trial question (experiment 3). Finally, 33-month-olds failed the low-response-generation-demand task when the object was not removed from the scene (experiment 4). Accordingly, the authors concluded that the data "provide strong support for the view that early difficulties with traditional falsebelief tasks stem from these tasks' processing demands" (p. 13364).

Opposing this nativist conclusion, we note that the modification according to which Ethan takes the apple away not only reduces the inhibition control demands but also primes children's attention to the correct answer. Indeed, after observing Emma placing the apple in the lidded box and leaving the room, children have clearly associated the apple with the container in which it was last. In addition, they have also associated Emma with the same container. In contrast, the saliency of the other container has progressively reduced: it is empty, and Emma has not recently interacted with it. Moreover, Ethan also interacts only with the container where Emma left the apple, and therefore his intervention does not disrupt these associations but possibly reinforces them. Thus, we propose that children could be solving the task by adopting an associationist and nonmentalistic strategy, which contrasts with the nativist view. 
Our proposal is enforced by the fact that the control conditions lacking in the LPDT constitute a methodological standard in the developmental literature (Southgate, Senju \& Csibra, 2007). It also resonates with a commentary to the initial study by Rubio-Fernández et al. (2017), who claimed that "children's training on factual 'where' questions might prompt them to point to the last location where the apple was." Whilst they proposed that children may apply behaviour-reading heuristics (Perner \& Ruffman, 2005), we argue that children's response may be driven by an attentional bias (e.g., Heyes, 2014). Nevertheless, because the analysis by Rubio-Fernández et al. (2017) is close to ours, we can discuss how Baillargeon and colleagues replied to the former to highlight the limits of their answer and bolster our point.

In addressing these concerns, Scott, Setoh, and Baillargeon (2017) argued that Rubio-Fernández' et al. (and likewise our similar interpretation) only account for the success but not the failure of children in the LPDT. Indeed, if children's answers really depended on an attentional bias, one should expect children of any age to select the perceptually more salient location significantly more often than the contrasting target (against evidence from experiments 2 and 3). However, we note, if - consistent with an information-processing model - the cognitive demands of processing the task question disrupt younger children's attention in the LPDT, they may also disrupt children's newly created associations between Emma, the apple, and the more salient container, thereby making it unsurprising that children answered at chance. In contrast, when the question prompt intervention relieves the cognitive burden of processing the task question, even younger children can maintain their understanding of the task scenario while answering the question. At that point, though, their response becomes subject to the attentional bias identified above. Thus, the reply by Baillargeon and colleagues rejects neither Rubio-Fernández' nor our analysis.

It may be counter-argued that our proposed solution is ad hoc, because it arbitrarily postulates that the associationist bias manifests only when the computational demands of the test question are sufficiently relieved by proper intervention. In replying, we note that the same sort of arbitrariness - if any - is also shared by the twofactor analysis that supports the nativist interpretation. To see this, consider again the discussion between the groups of Rubio-Fernández and Baillargeon. RubioFernández et al. (2017) noticed that reducing only the response generation demand did not improve children's success in the LPDT (experiment 4), while "if both inhibitory control and response generation are critical to passing a standard false-belief task, then reduced demands in either of these two processes ... should improve children's performance". In replying to this objection, Scott et al. (2017) argued that they had "never claimed that reducing either inhibitory-control or response-generation demands in a high-inhibition task should improve 2.5 -year-olds' performance ... [because] inhibitory-control and response-generation demands are not interchangeable and have different impacts on children's performance."

The problem with this response, however, is that no internal constraint of the information-processing model prescribes that the inhibitory-control and response generation demands of the traditional FBT are not interchangeable. Accordingly, if it is possible to tailor a theoretical interpretation to the empirical data - in the way that Baillargeon and colleagues suggest - to explain the attested asymmetry between the inhibitory-control and the response generation demands of the standard FBT, it is equally plausible to postulate that younger children's capacity to overcome the response generation demands in the LPDT is not interchangeable with the manifestation of the associationist attentional bias.

Concluding, we hope to have persuasively argued that our associationist proposal is at least as empirically grounded, methodologically valid, theoretically sound, and epistemologically justified as its nativist alternative. Accordingly, the data from the LPDT currently fail to support a nativist resolution of the debate on belief understanding in infancy.

\section{Acknowledgement}

We would like to thank Jedediah Allen, Federico Boem, Jeremy Carpendale, and Silvano Zipoli Caiani for useful discussion and comments on a previous version of this article.

\section{Funding Sources}

Duilio Garofoli was funded by the Gerda Henkel Foundation, grant code AZ 13/F/16, at the time of the development of this work.

\section{Author Contributions}

The order of the authors' names is arbitrary because this paper is fully collaborative. In compliance with specifications of the Italian Ministry of Education, Universities and Research, we clarify that Marco Fenici has written the first five paragraphs, and that Duilio Garofoli has written the remaining four. 


\section{References}

Baillargeon, R., Scott, R. M., \& Bian, L. (2016). Psychological reasoning in infancy. Annual Review of Psychology, 67(1), 159-186. https://doi. org/10.1146/annurev-psych-010213-115033

Grosso, S. S., Schuwerk, T., Kaltefleiter, L. J., \& Sodian, B. (2019). 33-month-old children succeed in a false belief task with reduced processing demands: A replication of Setoh et al. (2016). Infant Behavior and Development, 54, 151-155. https://doi.org/10.1016/j.infbeh.2018.09.012

Heyes, C. M. (2014). False belief in infancy: A fresh look. Developmental Psychology, 17(5), 649659. https://doi.org/10.1111/desc. 12148

Perner, J., \& Ruffman, T. (2005). Psychology. Infants' insight into the mind: How deep? Science, 308(5719), 214-216. https://doi.org/10.1126/ science.1111656
Rubio-Fernández, P., Jara-Ettinger, J., \& Gibson, E. (2017). Can processing demands explain toddlers' performance in false-belief tasks? Proceedings of the National Academy of Sciences of the United States of America, 114(19), E3750. https://doi.org/10.1073/pnas. 1701286114

Scott, R. M., Setoh, P., \& Baillargeon, R. (2017). Reply to Rubio-Fernández et al.: Different traditional false-belief tasks impose different processing demands for toddlers. Proceedings of the National Academy of Sciences of the United States of America, 114(19), E3751-E3752. https://doi.org/10.1073/pnas.1703665114

Setoh, P., Scott, R. M., \& Baillargeon, R. (2016). Two-and-a-half-year-olds succeed at a traditional false-belief task with reduced processing demands. Proceedings of the National Academy of Sciences of the United States of America, 113(47), 13360-13365. https://doi. org/10.1073/pnas.1609203113
Southgate, V., Senju, A., \& Csibra, G. (2007). Action anticipation through attribution of false belief by 2 -year-olds. Psychological Science, 18(7), 587-592. https://doi.org/10.1111/ j.1467-9280.2007.01944.x

Wellman, H. M., Cross, D., \& Watson, J. (2001). Meta-analysis of theory-of-mind development: The truth about false belief. Child Development, 72(3), 655-684. https://doi.org/10.1111/14678624.00304

Wimmer, H., \& Perner, J. (1983). Beliefs about beliefs: Representation and constraining function of wrong beliefs in young children's understanding of deception. Cognition, 13(1), 103-128. https://doi.org/10.1016/0010-0277 (83)90004-5 